\title{
International Journal of Medical Science and Health Research
}

Vol. 5, No. 05; 2021

ISSN: 2581-3366

\section{Assesment of Breast Cancer's Risk Factors in the Female Population of Kvemo Kartli (Georgia)}

\author{
Zurabashvili M ${ }^{1}$, PhD Kvanchakhadze R. ${ }^{1}, \mathrm{PhD}$ L. Baramidze ${ }^{2}$, PhD L.Gumbaridze ${ }^{2}$ \\ ${ }^{1}$ The University of Georgia, Faculty of Public Health 77a, M. Kostava str. Tbilisi, 0171, Georgia \\ ${ }^{2}$ Tbilisi State Medical University, Department of Public Health, Management Policy and \\ Economics 33, Vazha-Pshavela Ave.0186, Tbilisi, Georgia
}

doi: 10.51505/ijmshr.2021.5515

URL: http://dx.doi.org/10.51505/ijmshr.2021.5515

\begin{abstract}
The purpose of research was to study of the prevalence of breast diseases in women aged 35-65 in Kvemo Karti region, assessment of the epidemiological situation, identification of key risk factors. Research Goals were: Facilitation of early detection and prevention of breast cancer in Kvemo Kartli region; Identifying the link between the development of breast cancer and various risk factors; Reducing the burden of breast disease and mortality; Assessing access to and barriers to medical services to optimize and advocate for women's health; Cross-sectional research was held in several medical institutions in Rustavi with a high number of patients. The study population was women belonging to the 36-65 age group who have applied to a medical facility. The degree of correlation between risk factors and breast cancer was determined by bivariate analysis. There was a statistically significant correlation between the risk factors we assessed and the presence of breast cancer; the age of the respondents and breast cancer, more precisely, the chance of developing breast cancer is 4 times higher in the respondents over the age of 50; marital status and breast cancer, particularly in unmarried and widowed women, the chance of developing breast cancer is 3 times higher than in respondents with marriage and partner, between contraceptive use and breast cancer, particularly in respondents who use contraception, the chances of developing breast cancer are 9 times higher than in non-users. Early detection and prevention of mammary gland cancer by eliminating barriers to access to medical services and timely detection of breast diseases.
\end{abstract}

Keywords: Breast Cancer, Female Population, Oncological diseases, risk factors.

\section{Introduction}

Oncological diseases are a heavy burden on public health and are the second leading cause of death after cardiovascular disease, which is why the United Nations and the World Health Organization have made commitments in the recent years to improve prevention and treatments. Factors determining health, such as biological, physical, social and economic, affect cancer morbidity and prevalence. There is different data in different regions of the world on the prevalence of cancer and its risk factors. Social and economic inequalities between people and ethnic groups in terms of income, access to education, living conditions, employment and nutrition affect the cancer burden. Cancer mortality is strongly correlated with the stage of 


\section{International Journal of Medical Science and Health Research}

Vol. 5, No. 05; 2021

ISSN: 2581-3366

cancer diagnosis and the quality of treatment. People who are not able to see a doctor earlier due to unfavorable conditions or do not participate in screening programs have much higher rates of complications and mortality.

The most commonly diagnosed cancer profile in women is distinguished by its dichotomous nature - breast and cervical cancer. Breast cancer is the most common oncological disease in most countries. Breast cancer incidence is on the rise in many countries in Europe and Asia, according to GLOBOCAN Breast Cancer Age-Standardized Data, published in recent years.

In Georgia, the incidence of cancer among women of reproductive age is almost twice as high as the incidence in men; from the age of 60 and older, the incidence of cancer is higher in men. The most common five localized oncological diseases among women in 2019 were breast, thyroid, colorectal, the cervical or uterus cancers (4).

As for the age-related features of the most common oncological diseases among women, breast, Cervical or uterus cancers and cervical are in line with trends in other countries, although thyroid cancer is an exception, with its fairly widespread prevalence in the 20-35 age group. 15\% of breast cancer cases are genetic in nature. Numerous studies have been conducted around the world to study the role of genetic factors in the development of breast cancer. Based on scientific studies, it has been found that breast cancer pathologies, including breast cancer, are more prevalent in genotypically closed nations, such as the Georgian nation. Since the role of the genetic factor in the development of malignant breast cancer has been significantly elucidated, some studies have been introduced to examine the likelihood of detecting this pathology precisely at the gene level. In some countries, if a woman is found to have a mutation in the BRCA-1 and BRCA-2 genes on chromosomes 17 and 13, it is considered that she has a 99\% chance of developing breast cancer. The role of genetic factors is especially important if one has had a close relative with breast cancer, such as a mother or a sister, especially if the cancer was developed at a young age.

The epidemiology and the pathophysiology of postpartum breast cancer diseases are of interest for the transformation of existing international experience. Breast cancer associated with this factor develops during childbirth or within 1 year. The connection between childbirth and breast cancer is unknown. Immunological and endocrinological changes take place during pregnancy, leading to breast involution.

The negative effects of environmental factors, artificial abortions, refusal to breastfeed, taking oral contraceptives, irregular sex life are significant risks in the development of breast diseases and in particular cancer. Excess weight and obesity, lack of physical activity also have a significant impact on the development of pathological pathways. ${ }^{[1,2,5]}$

In case of breast cancer, screening allows for early detection of the diseases, while cancer treatment guarantees an increase in survival and reduction in mortality. In 2011, the state cancer screening program was introduced in Georgia, within the framework of which breast, cervical, colorectal cancer screening and prostate cancer management are carried out. ${ }^{[7,8]}$ 


\section{International Journal of Medical Science and Health Research}

Vol. 5, No. 05; 2021

ISSN: 2581-3366

There is no sharp variation in the incidence of cancer among the regions of Georgia, which excludes the existence of a cluster of oncological diseases in any region. No study has been conducted in any specific region of Georgia, which accurately recorded the risk factors for breast cancer. Kvemo Kartli is no expectation, in addition to the various factors specific to this region, which may have influence on the development of breast pathologies (existence of different ethnical groups, industrial production, characteristics of food, differences in culture, religion, access to health services, various endemic diseases, etc.). Despite ongoing long-term research, treatment and prevention activities, breast diseases are a major public health problem throughout Georgia and in each of its region. Thus, the study of breast pathologies and, in particular, cancer risk factors in Georgia in female population of Kvemo Kartli is especially important and useful in the timely detection and prevention of breast cancer and other diseases.

\section{Reasons for Research:}

Study of the prevalence of breast diseases in women aged 35-65 in Kvemo Karti region, assessment of the epidemiological situation, identification of key risk factors.

\section{Research Goals:}

- Identifying the link between the development of breast cancer and various risk factors;

- Assessing access to and barriers to medical services to optimize and advocate for women's health;

\section{Methods}

Cross-sectional (prevalence) research was held in several medical institutions in Rustavi with a high number of patients. Given that, according to the World Health Organization, the risk of developing breast cancer increases with age and reaches its highest rate between ages of 60-69, the study population was women belonging to the 36-65 age group who have applied to a medical facility from January to June 2021.

Sample Size: the study population consists of women aged 35-65 living in Kvemo Kartli region. The sampling frame is based on the Georgian Census Database. Considering that 85294 women in the indicated age group live in the Kvemo Kartli region and, according to the literature, the prevalence of breast cancer in the female population is $15 \%$, the error in the formula used to determine the $95 \%$ reliability $(\mathrm{Z}=1.96)$ was set at $5 \%(\mathrm{e}=0.05)$, the prevalence of the main indicators was considered to be $15 \%(\mathrm{P}=0.15)$; Design effect -1.5 , response rate $80 \% .194$ respondents were defined as the required sample size $\left(n=Z^{2} P(1-P) / e^{2}\right)$. Given that breast pathologies are correlated with a number of factors (age, marital status, family history of disease, radiation and medical treatment, social and economic conditions, etc.), the questionnaires include all possible risk factors. Research Tools was standard questionnaires, in which questions, in addition to the risk factors for breast pathologies were related to the demographic characteristics, treatment provided, access to medical services, barriers, etc. Respondents were interviewed by a doctor using a specially designed questionnaire. The study did not share any personal 


\section{International Journal of Medical Science and Health Research}

Vol. 5, No. 05; 2021

ISSN: 2581-3366

information of respondents. Prior to the survey, the importance of the survey was explained to the respondents and each of them has signed a consent to participate in the survey.

Database analysis was carried out using strategic and bivariate research methodology (using statistical package Epi-info 7). The bivariate analysis calculated prevalence rations between risk factors and breast cancer (using 95\% confidence interval).

\section{Results}

Research was conducted in Rustavi, in several medical institutions distinguished by high turnout of Rustavi patients. The study population is women aged 35-65, who have been diagnosed with various breast disease. 194 respondents were involved in the study. Out of the 194 participants, the largest age group was 50-59 (33\%), and the biggest ethnical groups were Georgians (120, $(60 \%)$ ), and Azerbaijani (58, (30\%)). The majority of women surveyed were married, 4\% unammaried, 5\% divorced, 9\% widowed, etc. The majority of respondents have secondary education, many of whom, especially Azerbaijani women living in rural areas are housewives. $45 \%$ of respondents are uninsured, of which $64 \%$ have private and $36 \%$ state insurance.

$23 \%$ of respondents use contraceptives. Respondents who used contraceptives were 9 times more likely to develop breast cancer.

One of the goal of study was to establish a relationship between the menstrual cycle onset age, number of birth and abortions to breast disease. According to available literature, women that start their menstrual cycle late, have a higher number of births, do not lactate or have a short menstrual cycle have a higher risk of breast disease and breast cancer. $85 \%$ of respondents started their menstrual cycle between the ages of 11-13, while $15 \%$ started between the ages of 14-16. $75 \%$ of respondents were breastfeeding at some point of their life. $88 \%$ of the respondents have experienced childbirth, and $63 \%$ had experienced an abortion, out of which $88 \%$ have experienced an artificial abortion, while $25 \%$ have experienced a natural abortion. According to data, 58 respondents (55\%) have terminated 1-3 pregnancies, 39 respondents (37\%) have terminated 4-5 pregnancies, while 8 respondents (8\%) have terminated 6-10 pregnancies (3).

Diagram \#1. Distribution of Respondents by the Number of Pregnancies

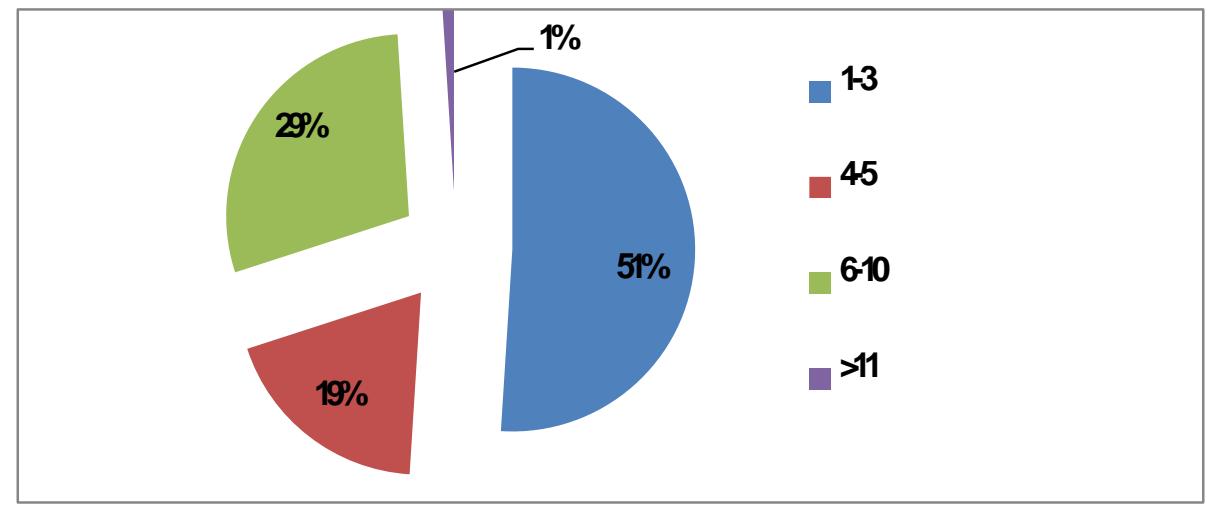

www.ijmshr.com

Page 119 
Diagram \#2. Distribution of Respondents by the Number of abortions

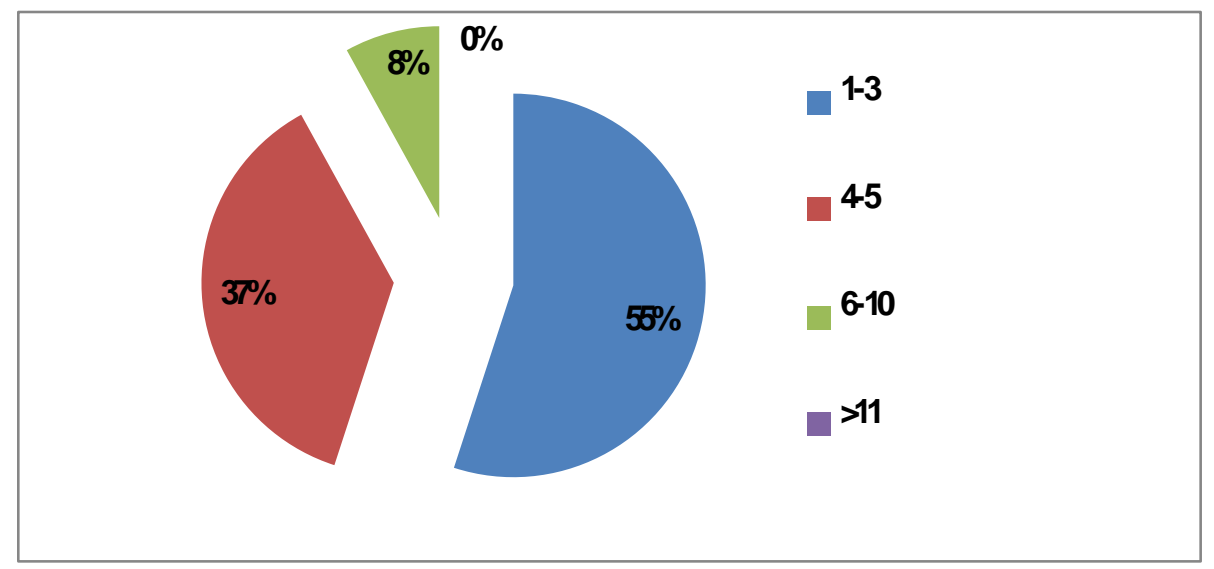

There was not statistically significant correlation between the age of onset of menstrual cycle, the number of birth and the number of abortions and development of breast disease.

According to the literature sources, the age of menopause has an impact on the forming of the breast cancer. In the Female Population of Kvemo Kartli the ratio of the respondents who were in the period of menopause is $43 \%$, out of which $69 \%$ claimed that their menstrual cycle ended while being $45-50$ years old, $31 \%$ claimed that it ended when they were 51-55 years old. There is no correlation towards this approach either (6).

During the last 2 years $87 \%$ of the respondents indicated the pain in the breasts, $44 \%$ indicated the tightness in the breast and $8 \%$ claimed they had a bloody discharge out of their breasts.

In the area of the mammary gland pathologies the highest rate of $37 \%$ had the diffuse fibro adenomatous mastopathy, the next was the cancer of the mammary gland with the rate of $25 \%$.

Diagram\#3. The Dustribution of the Mammary Gland Pathologies

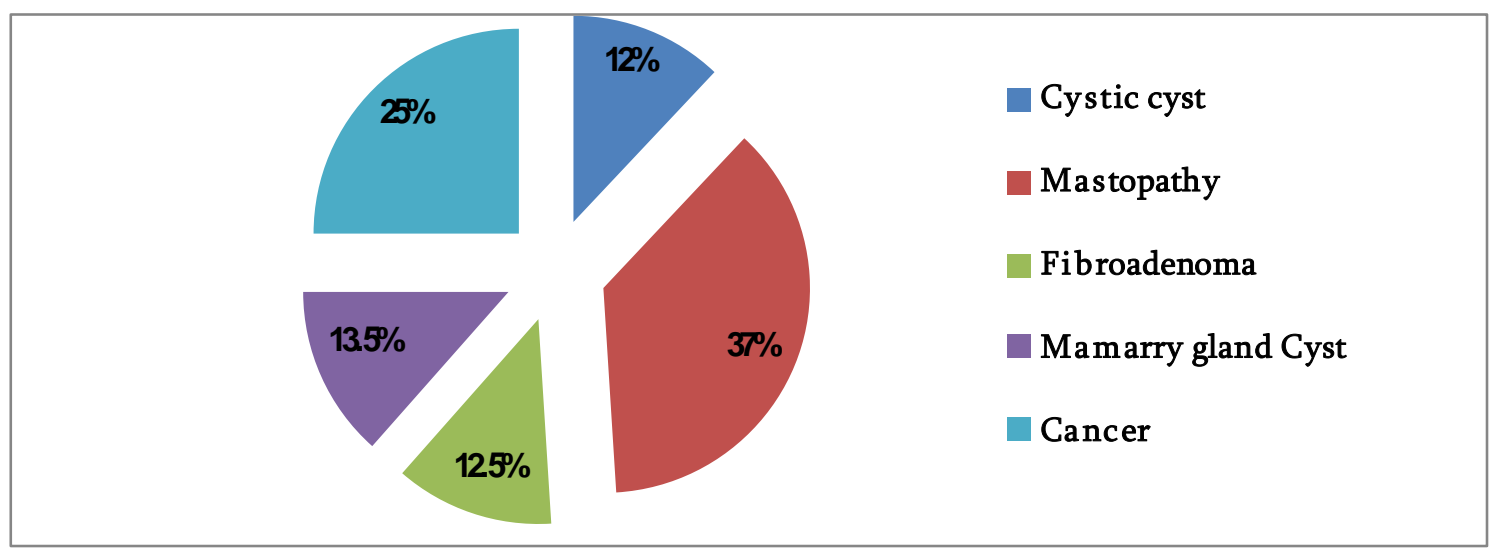




\section{International Journal of Medical Science and Health Research}

Vol. 5, No. 05; 2021

ISSN: 2581-3366

The distribution of the mammary gland pathologies in the 35-65 years old women in Kvemo Kartli showed the following results: The highest rate of the diffuse fibrous-cystic mastopathy (24\%), the fibroadenoma of the mammary gland (28\%), breast cyst (24\%) were in the women of 35-39 years old. The cases of diffuse fibroadenoma tous mastopathy grew with the age increase until 60-65 years when it started to decrease. The rate of the cancer of the mammary gland increased with the age until its maximum 60-65 years. The last result shows the correlation between the respondents' age and the breast cancer, more precisely, the probability of forming the mammary gland cancer was 4 times bigger for the respondents aged 50 or more.

Table \#1.The Distribution of Mammary Gland Pathologies Given the Age Group

\begin{tabular}{|c|c|c|c|c|}
\hline Disease & Aged 35-39(\%) & Aged 40-49(\%) & Aged 50-59(\%) & Aged 60-65(\%) \\
\hline $\begin{array}{c}\text { Diffuse fibrous-cystic } \\
\text { mastopathy }\end{array}$ & $24 \%$ & $13 \%$ & $7 \%$ & $2 \%$ \\
\hline $\begin{array}{c}\text { Diffuse fibroadenoma tous } \\
\text { mastopathy }\end{array}$ & $20 \%$ & $41 \%$ & $52 \%$ & $35 \%$ \\
\hline $\begin{array}{c}\text { Mammary gland } \\
\text { fibroadenoma }\end{array}$ & $28 \%$ & $13 \%$ & $2 \%$ & $5 \%$ \\
\hline Mammary gland cyst & $24 \%$ & $15 \%$ & $7 \%$ & $7 \%$ \\
\hline Mammary gland cancer & $4 \%$ & $18 \%$ & $32 \%$ & $51 \%$ \\
\hline All & $100 \%$ & $100 \%$ & $100 \%$ & $100 \%$ \\
\hline
\end{tabular}

$63 \%$ of the respondents are being treated for the mammary gland diseases.

Table \#2. The Distribution of the Types of the Treatment Method for the Mammary Gland Pathologies

\begin{tabular}{|c|c|}
\hline Mammary Gland Pathologies & Quantity \\
\hline Medicinal & 20 \\
\hline Surgical & 65 \\
\hline Radiotherapy & 33 \\
\hline Phytotherapy & 59 \\
\hline All & 177 \\
\hline
\end{tabular}

Some of the patients used couple of treatment methods together.

Table \#3. The Distribution of the Mammary Gland Diseases and the Treatment Method

\begin{tabular}{|c|c|c|c|c|}
\hline Disease & $\begin{array}{c}\text { Medicinal } \\
(\mathbf{\%})\end{array}$ & $\begin{array}{c}\text { Surgical } \\
(\mathbf{\%})\end{array}$ & $\begin{array}{c}\text { Radiotherap } \\
\mathbf{y}(\mathbf{\%})\end{array}$ & $\begin{array}{c}\text { Phytotherap } \\
\mathbf{y}(\mathbf{\%})\end{array}$ \\
\hline Diffuse fibrous-cystic mastopathy & & & & $19 \%$ \\
\hline Diffuse fibroadenoma tous mastopathy & & & & $61 \%$ \\
\hline Mammary gland fibroadenoma & $5 \%$ & $29 \%$ & & $3 \%$ \\
\hline Mammary gland cyst & $5 \%$ & $3 \%$ & $3 \%$ & $14 \%$ \\
\hline Mamary gland cancer & $90 \%$ & $68 \%$ & $97 \%$ & $3 \%$ \\
\hline All & $100 \%$ & $100 \%$ & $100 \%$ & $100 \%$ \\
\hline
\end{tabular}




\section{International Journal of Medical Science and Health Research}

Vol. 5, No. 05; 2021

ISSN: 2581-3366

The rate of the respondents who claim that there was one or couple reasons for delaying the manifestation of the mammary gland pathologies is $91 \%$ (174). Among them, economic reason should be noted, which is in connection with the Covid-19 pandemic (social isolation). The second most common reason was psych emotional fear, a portion of respondents in study were not screened on time as they had some kind of fear as well as considering the fact that the majority of the patients in study live in the villages, the availability of the transportation to the healthcare service centers are low because most of the time the respondents depended either on the spouses or on other family members. It should also be noted that $28 \%$ of them had language barriers. These factors interfere with the participation of the respondents in the screening program, treatment on time and the actions that could prevent the diseases in the first place.

Table \#4. Distribution of respondents according to the reasons hindering the timely detection of breast pathology

\begin{tabular}{|c|c|c|}
\hline Barriers & Quantity & Percentage \\
\hline Geographical & 12 & $19 \%$ \\
\hline Linguistic & 49 & $28 \%$ \\
\hline Economical & 131 & $76 \%$ \\
\hline Religious & 27 & $16 \%$ \\
\hline Psycological fear & 104 & $59 \%$ \\
\hline
\end{tabular}

The breast cancer had 25\% of the respondents out of which $67 \%$ were $51-65$ years old. It is general knowledge that the unfavorable environmental conditions have a negative impact on the mammary gland pathologies, prolonged working in the unhealthy industrial environment may increase the chances of developing breast pathologies, however, most of patients were rural and their main activities involved agricultural work. One of the most important risk factors for the development of breast pathologies is nutritional characteristics and overweight, in the last decade, obesity rates have increased in all countries due to low physical activity and unhealthy diet; Obesity is already responsible for $6 \%$ of breast cancer cases. For this reason, we used a questionnaire to determine the eating patterns of the respondents as well as the types of the food they consumed, more precisely, we determined whether they had been mixed, vegetarian, vegan or pescatarian (vegetarian that consumes seafood) eaters. Most of the women were fed fatty meats regardless of nationality and age and did not include seafood and fruits and vegetables in their diet, only in small quantities, this is consistent with the Steps survey data. It is known from modern literature that the consumption of tobacco and alcohol affects the development of breast diseases. Out of all respondents $12 \%$ consumed tobacco (7.5\% of them urban and $3.5 \%$ rural) and $1 \%$ periodically drank alcohol. Consequently, these data do not provide a basis for linking the risks of developing various breast diseases for the respondents included in study to tobacco and alcohol. 


\section{The Results of the Analytical Research}

The degree of correlation between risk factors and breast cancer was determined by bivariate analysis. There was a statistically significant correlation between the risk factors we assessed and the presence of breast cancer:

1. There was correlation between the age of the respondents and breast cancer, more precisely, the chance of developing breast cancer is 4 times higher in the respondents over the age of 50 , the odds ratio $(\mathrm{OR})=4.495 \% \mathrm{CI}(2.2-8.8)$,

2. There was correlation between marital status and breast cancer, particularly in unmarried and widowed women, the chance of developing breast cancer is 3 times higher than in respondents with marriage and partner $(\mathrm{OR})=3.395 \% \mathrm{CI}(1.5-7.2)$,

3. There was correlation between contraceptive use and breast cancer, particularly in respondents who use contraception, the chances of developing breast cancer are 9 times higher than in non-users $9.995 \%$ CI (2.3-42.6),

Fig. \#4. The prevalence of the odds ratio $(\mathrm{OR})$ between the respondents (of $>50)$ and the breast cancer; between marital status (unmarried and widowed) and breast cancer; between contraceptive use and breast cancer

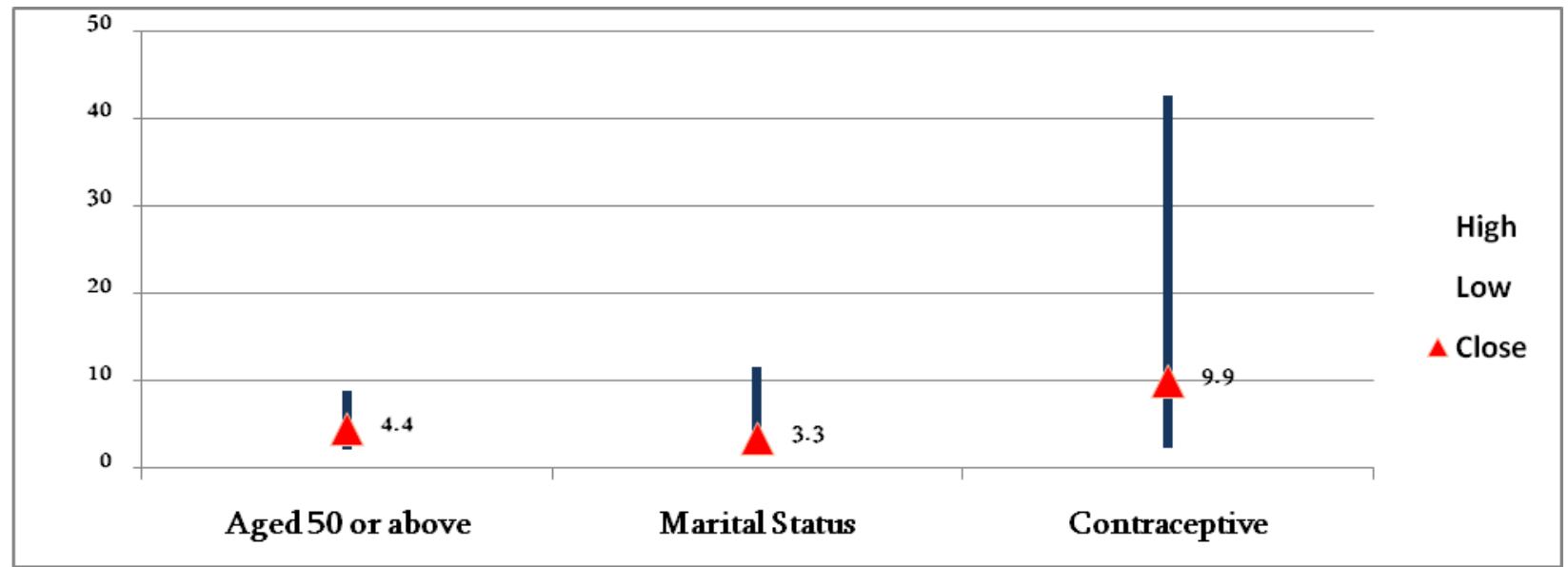

\section{Discussion}

Study found that in Kvemo Kartli, $25 \%$ of respondents had breast cancer, $67 \%$ of whom were 51-65 years old. A correlation between breast cancer and oral contraceptives has been shown, which is in line with other research ( $\mathrm{Ji}, \mathrm{Li}-\mathrm{Wei}$ Mdaand co-avt), especially if patients started taking oral contraceptives at an early age(1). According to Soheila Ehsanpour (and co-avt), taking oral contraceptives for 36-72 months increases risk of breast cancer 2,18 times. The increased risk was not connected contraceptive usage habits (5). In other words, usage of oral contraceptives in any amounts increased risk of breast cancer in every age group. The connection between usage of contraceptives and developing breast cancer in Kvemo Kartli region has not been studied in such detail (taking into account age and usage time). 


\section{International Journal of Medical Science and Health Research}

Vol. 5, No. 05; 2021

ISSN: $2581-3366$

According to the American Cancer Society, about 1 out of 8 invasive breast cancers develop in women younger than 45. About 2 out of 3 invasive breast cancers are found in women 55 or older (9). In addition, in the female population of Kvemo Kartli residents statistically significant correlation of breast cancer is 4 times higher in the respondents over the age of 50 .

According to the American Cancer Society early menstrual periods before the of age 12 and starting menopause after age 55 expose women to hormones longer, raising their risk of getting breast cancer. Women who have a higher number of births, do not lactate or have a high number of abortions have a higher risk of breast disease and breast cancer (9).

No statistically significant correlation was found in the relationship between the development of breast cancer and these risk factors, since the study was conducted on a relatively small group, it is advisable to further study this relationship in a relatively large population group.

\section{Conclusion}

Thus, in the population of women in Kvemo Kartli, identifying the connection between the mammary gland diseases, in particular breast cancer, and the important risk factors can help both physicians and patients to take decisive action in the development of personalized medicine. Early detection and prevention of mammary gland cancer by eliminating barriers to access to medical services and timely detection of breast diseases.

\section{References:}

Ji, Li-Wei MDa; Jing, Chun-Xia MDa; Zhuang, Su-Lian MDa; Pan, Wei-Cheng BDb; Hu, XingPo BDc, $*$ Effect of age at first use of oral contraceptives on breast cancer risk Medicine: September 2019 - Volume 98 - Issue 36 - p e15719, doi: 10.1097/MD.0000000000015719

Mary Murithi, Teresa Kerubo, OgetoTeresa, Kerubo Ogeto, Michael Walekhwa, Oral contraceptives and intrauterine devices as risk factors for breast and cervical cancers: a systematic review June 2020

Rossana Ruiz 1, Carmen Herrero 2, Kathrin Strasser-Weippl 3, Diego Touya 4, Jessica St Louis 5, Alexandra Bukowski 6, Paul E Goss 7Epidemiology and pathophysiology of pregnancy-associated breast cancer: A review, PMID: 28732325 DOI: 10.1016/j.breast.2017.07.008.

Shibitidze R.Kipiani, E.Prevalence, Risk and Management of Breast Cancer https://www.caucasushealth.ge 1E ISSN 2449-2450 Caucasus Journal of Health Sciences and Public Health, Volume 1, Supplement 2, June 2017

Soheila Ehsanpour 1, Fahime Seyed Ahmadi Nejad, Fariborz Mokarian Rajabi, Fariba Taleghani Investigation on the association between breast cancer and consumption patterns of combined oral contraceptive pills in the women of Isfahan in 2011, Iran J Nurs Midwifery Res. 2013 May;18(3):186-90. 
Torgny Karlsson 1, Therese Johansson 1 2, Julia Höglund 1, Weronica E Ek \# 1, Åsa Johansson \# 3Time-Dependent Effects of Oral Contraceptive Use on Breast, Ovarian, and Endometrial Cancers2021 Feb 15;81(4):1153-1162. doi: 10.1158/0008-5472.CAN-202476. Epub 2020 Dec 17

Vashakidze N, Mebonia N, Kereselidze M, Gvamichava R, Zhizhilashvili S, EFFECT OF SELECTED PROGNOSTIC AND RISK FACTORS ON SURVIVAL OF WOMEN WITH BREAST CANCER IN GEORGIA, Georgian Med News. 2018 Jun;(279):23-28.

Vashakidze N, Mebonia N, Gvamichava R.EFFECT OF AGE AT DIAGNOSIS ON THE PROGNOSES IN FEMALE BREAST CANCER PATIENTS IN GEORGIA.Georgian Med News. $\quad 2018 \quad$ Nov;(284):27-32.PMID: 30618384 https://www.cancer.org/cancer/breast-cancer.html 